GPO PRICE

CFSTI PRICE(S)

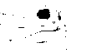

Hard copy $(\mathrm{HC}) \frac{2.00}{.50}$

ff 653 July 65

\title{
RELATIVE ADVANTAGES OF SMALL AND OBSERVATORY TYPE SATELLITES
}

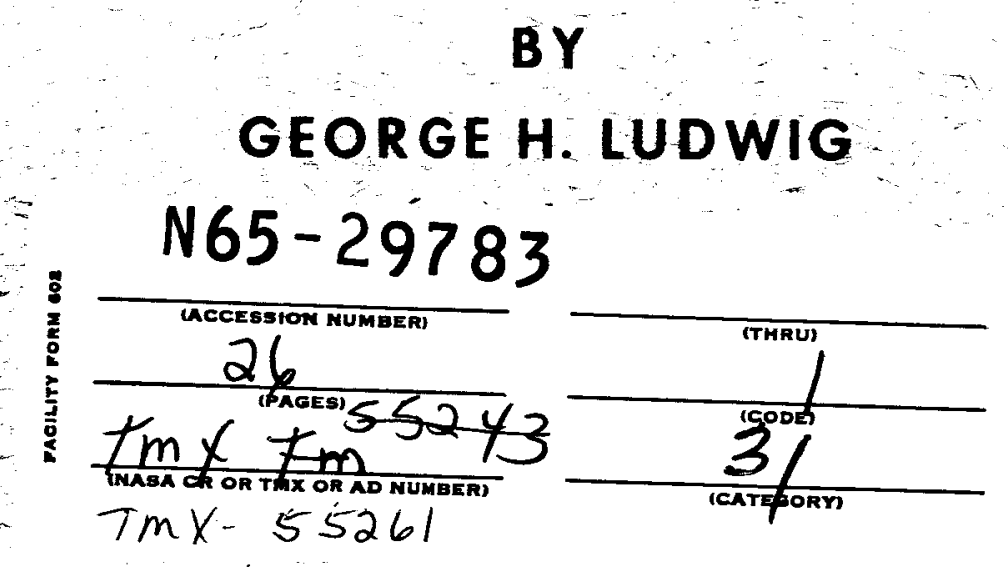

MAY 1965

NASA

GODDARD SPACE FLIGHT CENTER GREENBELT, MARYLAND

Energetic Porticles Preprint Series 


\section{RELATIVE ADVANTAGES OF SMALL AND OBSERVATORY TYPE SATELLITES*}

by

George H. Ludwig

May 1965

Space Sciences Division NASA/Goddard Space Flight Center Greenbelt, Maryland, USA

* Prepared for presentation to the COSPAR Symposium, Buenos Aires, Argentina, 13-19 May is65. 
RELATIVE ADVANTAGES OF SMALL AND

OBSERVATORY TYPE SATELLITES

by George H. Ludwig

NASA/Goddard Space Flight Center

Greenbelt, Maryland

\section{ABSTRACT}

29783

Both the relatively small Explorer and the large Orbiting Observatory classes of scientific satellite have advantages which need to be considered carefully when a new space experiment is to be performed. The small satellite offers greater choice in tailoring the orbit to the experiments. The orbital, orientation, telemetry, and operational needs of a particular experiment are not usually compromised to as large an extent because fewer experiments are involved. The smaller size simplifies the electrical, magnetic, and radiated interference problem, since fewer operating components are involved. It provides greater ease in testing and scheduling, and permits a shorter pre-launch lead time.

The larger observatory permits the conduct of more complex or larger numbers of related experiments for the more detailed study of the co-relationships between the numerous space phenomena. Since it is less highly integrated, standard experiment/spacecraft interfaces can be defined to simplify the experiment design and integration problems. The larger size permits the use of higher capacity and more flexible data systems and more precise active orientation systems. Operational efficiency is higher, since the data from a large number of experiments can be recorded and processed simultaneously. It is concluded that both types should continue to be used to meet the varied requirements of the space sciences program.

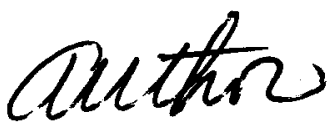


INTRODUCTION

The earth satellites presently being used for space science investigations can be grouped into two broad classes. The first is the relatively small satellite typified by the Explorer series. It includes the Explorer, Vanguard, Solar Radiation, Injun, Traac, Starad, Lofti, Rados, and International Program (Ariel, Alouette, and San Marco) satellites. The second class consists of the larger observatories, and includes the Orbicing Solar Observatories (OSO), Orbiting Geophysical Observatories (OGO) and Orbiting Astronomical Observatories (OAO). An Advanced Orbiting Solar Observatory (AOSO) is also being developed. The relative merits of the two classes have been the subject of many debates. This paper is an attempt to summarize some of the more significant advantages of each type. An attempt to evaluate the advantages of the two classes is especially timely, since the second and third observatories (OGO-I and OSO-II) have been launched, and it is becoming possible to discuss statistically significant actual experience and performance. To illustrate some of the arguments, reference will be made to several specific satellites. The Interplanetary Monitoring Platform (IMF-I, 1963-46A, or Explorer XVIII) typifies the small Explorer class satellite built within a NASA laboratory. A satellite built at another government laboratory is typified by the Naval Research Laboratory Solar Radiation satelite (1964-01D). The University of Iowa Injun series is the only presently existing sateliite series built entirely at a university laboratory, and will be represented by Injun IV

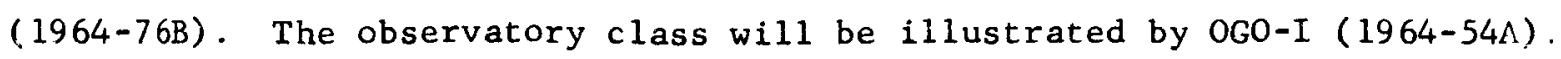




\section{WEIGHT AND VOLUME CAPABILITY}

The Explorer class satellite weights have ranged from $7 \mathrm{~kg}$ for the atmospheric drag balloon (Explorer IX) to $14 \mathrm{~kg}$ for the early energetic particles Explorers, to $184 \mathrm{~kg}$ for the Atmospheric Structures Explorer XVII. Onıy chree have weighed more than $120 \mathrm{~kg}$ as is shown in figure 1 . The observatory weights have ranged from $208 \mathrm{~kg}$ for $0 S O-I$ to $488 \mathrm{~kg}$ for Oro-I. The first OAO will weigh about $1600 \mathrm{~kg}$. Weight has historically been the primary limiting factor when choosing the experiments for each spacecraft mission because of launch vehicle limitation. The large weight carrying capability of the observatories becomes especially significant:

1. if individual experiments are very heavy

2. if it is necessary to conduct a large number of experiments at the same position in space at the same time to correlate various space phenomena.

Volume for experiments has always been secondary in importance, and will probably continue to be so, except for experiments requiring large optical systems or apertures. Presumably, these large optical, near optical, and radio astronomy experiments can be performed only on the larger observatories.

On the other hand, large weight and volume may be a liability for some experiments. When searching for relatively rare elementary particles, the large mass of an observatory can create an unacceptable background flux of secondary particles. A large volume may complicate the outgassing problem, causing a local contamination for atmospheric and ionospheric experiments. 
ATTITUDE CONTROL

Numerous experiments require some form of attitude control. Some of these requirements can be met on both small and large spacecraft, while others are more feasible with one class or the other.

There are many possible types of attitude control, each having interest for certain classes of experiments.

1. Non-oriented and random orientation. Some experiments are intended to observe an isotropic field, and therefore require no orientation. It is possible to perform some measurements of a non-isotropic field if the instantaneous orientation of the non-oriented spacecraft is knowr. Other experiments may actually prefer a random tumble to obtain data statistically integrated over the entire sphere. These missions are best performed by small satellites, since all sets of experiments flown on larger observatories will almost always contain sub-sets which require orientation. In addition the problems of providing adequate solar power and acquiring data telemetered to the earth from tumbling spacecraft would be more serious for the larger observatories, where higher capacity data systems are normally employed.

2. Spin orientation. Spin orientation is acceptable or preferable for many experiments, and is easy to achieve for small sateilites, since most of the smalier unguided final rocket stages require spinning for stability. A separate spin subsystem would have to be added for the observatories. Observatories can be made to spin satisfactorily as demonstrated by the actual operation of OGO-I. 
3. Earth orientation. Earth orientation is desired for many spacecraft to permit use of high gain antennas for high capacity data transmission; and some experiments requixe earth orientation (either toward or away from the earth). Some techniques, such as gravity gradient stabilization, can be used on either large or small satellites. Others, such as the use of horizon scanners and active torquers of various types, require use of the larger observatories because of their complexity. This results from the fact that active attitude control system weights do not, in general, scale linearly with total spacecraft weights. The smallest active earth orientation system has a weight which is too large for the smaller satellites.

4. Magnetic field orientation. Alignment of sensors at an angle fixed relative to the local magnetic field is desirabie for certain classes of experiments designed to investigate the low energy particles whose motions are controlled by the earth's magnetic field. Such experiments can be performed near the earth most easily on small satellites, since the controlling torques are small and can be produced directly by the magnetic field, as was done on the Injun satellites. If experiments of this type are to be performed at higher altitudes, where the earth's field is weaker, active attitude control systems using sensitive magnetic field sensors and active torquing devices would De uecessary. The active system would probably require a larger satellite. Actually, these experiments can be performed at the cost of somewhat increased instrumentation and data processing complexity, by appropriate sampling of scanning detectors, if the direction of the field is measured concurrently. 
5. Orientation with respect to the direction of motion is . desirable for experiments which investigate particles whose velocities are lower than or comparable with that of the spacecraft. Of course, some of these experiments can be performed on spinning satellites of all sizes by the use of appropriate time sampling techniques But continuous orientation of the sensors along the satellite velocity vector or in the orbital plane requires the use of an active orientation system in a larger satellite.

6. Sun orientation. Sun orientation is desirable to simplify the collection of solar energy by solar cells or reflectors, and many experiments require solar orientation. A few solar orientation systems, such as a torqued spin axis system, are suitable for use on small sate1lites. And some solar experiments can be performed by careful timing of the observations from a spinning satellite. It is likely, however, that solar experiments requiring medium to high pointing accuracy or solar disc scanning will continue to be located on the larger observatories to take advantage of their higher capability orientation system.

7. Celestial inertial orientation. Many astronomical experiments require orientation toward a fixed point on the celestial sphere, and a capability for moving to a new point between observations. Spin stabilized small satellites are acceptable for some experiments requiring low pointing accuracy, for example, gamma ray astronomy, where the sources are very weak and integration over a large solid angle is necessary. Most of these experiments, however, require an active orientation system and, therefore, the larger observatories. 
8. Combinations of several different orientation schemes lead, in general, to greater complexity and larger spacecraft. The OSO is spin and sun oriented. The OGO employs earth, sun, and orbit plane orientation, while OAO will contain a highly accurate directable celestial inertial system and a low accuracy sun orientation system.

In summary, passive orientation techniques are useful on small satellites, and some of them are better suited to small satellites than large ones. A few simple active systems are being used or considered for use on small satellites. For example, Iowa has used an active magnetic field orientation system on Injun. Any but the simplest active system requires the use of larger observatories.

DATA HANDLING, STORAGE, AND TELEMETRY

As in the case of the active attitude control systems, data handing, storage, and telemetry systems do not scale linearly with spacecraft weight Doubling the number of time multiplexer inputs, for example, requires only one additional stage in the multiplexer counter. In addition, the capability of electronics equipment increases roughly with volume, or the cube of linear dimensions, while the container and other structural weight increases more nearly as the surface area, or the square of linear dimensions. Therefore, doubling the data handling system weight permits more than doubling the number of functional circuits. For these reasons, the capability of the data system per pound of experiments tends to be larger for larger spacecraft.

Experiments in space are designed to measure fields which may be functions of time and position from a location which is, in turn, moving. 
The ability to make a meaningful mapping of that field depends almost . entirely on the information bandwidth, assuming that adequate freedom in selection of sampling formats and times exist. With present data systems, this freedom usually exists in both large and small satellites. To illustrate, figure 2 shows the telemetry format for IMP-I and figure 3 shows the format for the main commutator in 0GO-I. In addition to this main commutator, OGO has a 128 word sub-commutator for slowly varying measurements and a flexible format commutator for a relatively few rapidly varying measurements. Both IMP and OGO are able, by proper assignment of the many telemetry words to the various experiments, to meet a very large number of the sampling sequence needs.

Thus, the ability of an experiment to effectively map a field depends most directly on the information bandwidth available for that experiment. Table $I$ is a tabulation of the bandwidth per experiment and bandwidth per unit experiment weight for several representative satellites. It can be seen that, from the standpoint of the data system alore, it is presently possible to perform a more comprekersive and detailed mapping of a property of space on the larger spacecraft.

\section{POWER}

The total amount of solar power obtained on a satellite is not a strong function of satellite size. Power available from an array is very nearly a linear function of both array area and weight. Non-oriented satellites require more area to obtain a given power because the sun does not always illuminate the array normally. On the other hand, the large actively oriented satellites require electrical power to keep the arrays 
岂命

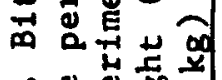

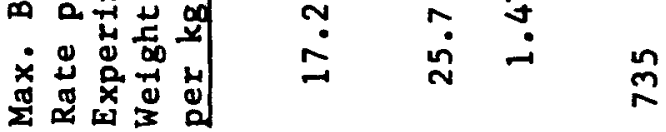

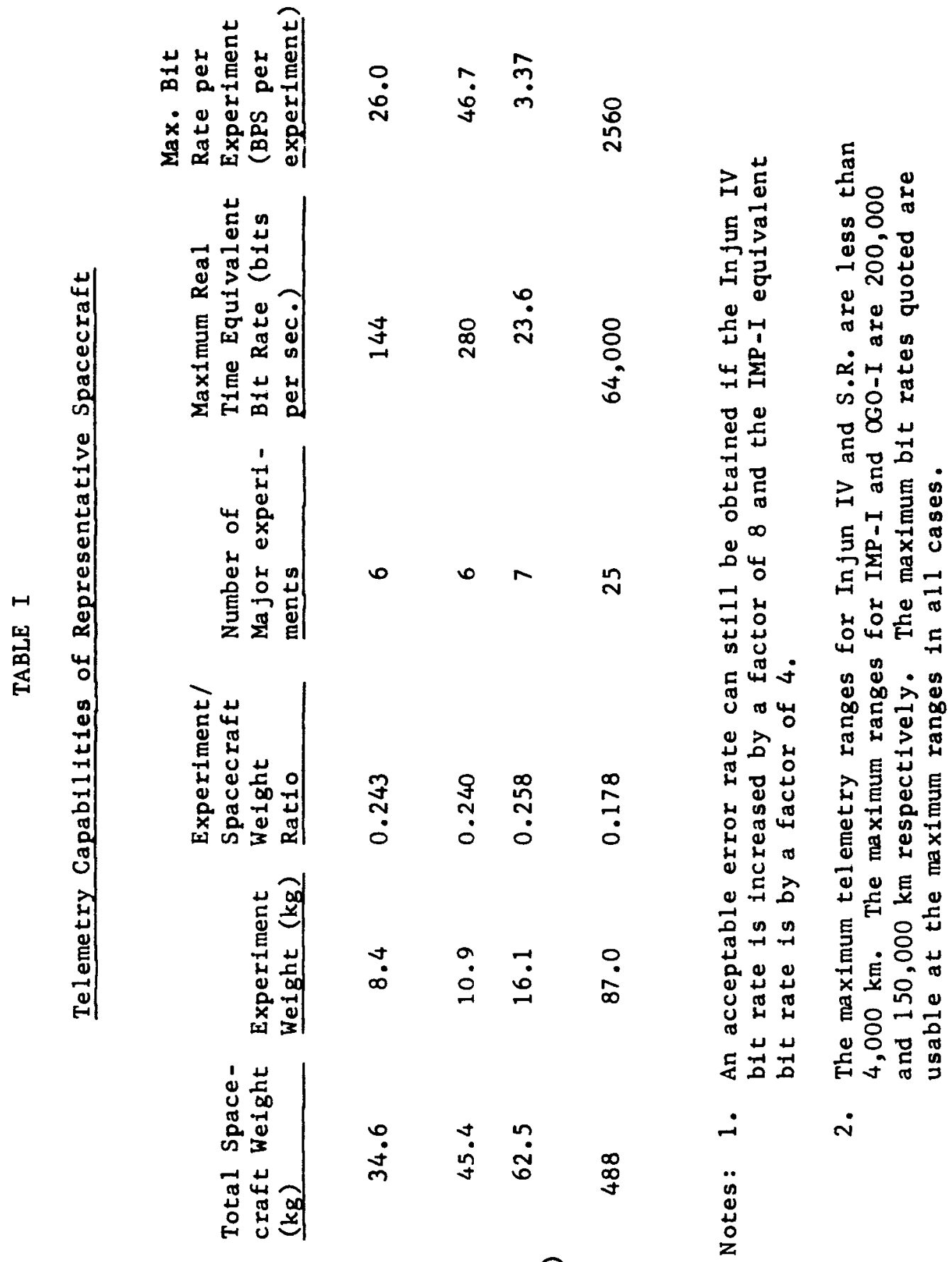

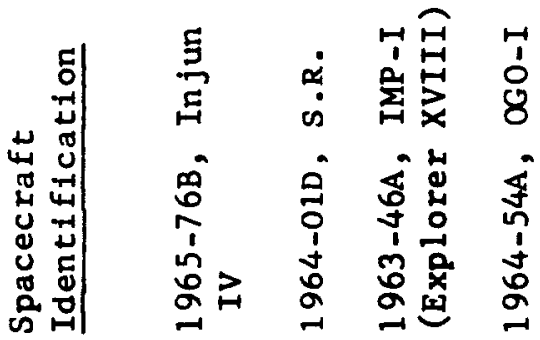


directed toward the sun. Table II shows the power available to experiments in the four representative spacecraft.

Since power for long periods of operation has always been expensive in terms of spacecraft weight, considerable effort has been expended to design electronic circuits to operate on very low power. The same iechniques have been employed on satellites of all sizes.

TABLE II

Experiment Power on Representative Spacecraft

\begin{abstract}
Spacecraft Identification
\end{abstract}

1965-76B, Injun IV

1964-01D, S.R.

1963-46A, IMP-I

1964-54A, or:0-?

\begin{abstract}
Total Spacecraft. Power (Watts)
\end{abstract}

5.97

3.39

38.0

260.0

\section{Experiment} Power (W)

2.89

1.89

9.68

50.0

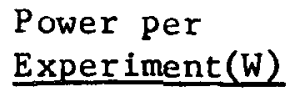

0.482

0.315

1.38

2.40
Power per Experiment Weight (W per kg)

0.344

0.174

0.601

0.690

\section{INTERFERENCE TO EXPERIMENTS}

Many experiments are susceptible to interference from other experiments and from the spacecraft. This includes interference in many forms, such as electrical interference from oscillations and transients in operating systems, magnetic interference from ferromagnetic materials and electrical current loops, mechanical interference from moving masses, radioactive interference from calibrating sources, particle interference from secondary interactions of cosmic rays in the mass of the spacecraft, 
and interference from the gases released from the spacecraft. Obviously, the smaller the spacecraft and the fewer the experiments, the more manageable is the interference problem. Interference is combated on the larger observatories where many diverse experiments are carried by the use of booms to isolate detectors from the rest of the observatory, and by careful interference engineering and control. With great care, the interference levels on the larger observatories can be made acceptable for a large number of experiments. Some of the most sensitive ones, however, may have to be performed on special small satellites.

\section{PROGRAM COORDINATION AND EASE OF INTEGRATION}

Small satellites are presently easier to coordinate and integrate, primarily because of the smaller number of individuals involved. The amount of this effort which experimenters are obliged to perform varies considerably, and is influenced strongly by the quality of the design and specification of the electrical, mechanical, and thermal interfaces between the experiments and spacecraft. It is also affected by the interfaces between the operations and data processing groups and the experimenters. Standardization and careful specification of these interfaces before experiment design begins greatly simplifies the coordination, integration, and operations efforts. In principle, the standard observatory concept could lead to a significant reduction in the liaison requirements, since the same observatory and operational systems are used repeatedly, permitting careful specification and development of familiarity with the system by the experimenters. The smaller spacecraft are unable to achieve this goal because of the continuing pressure to specialize the 
spacecraft to better meet the needs of the experiments. It is common. on these spacecraft to design the experiments, spacecraft, and data processing systems concurrently, so that the interfaces evolve in the process of the work. This leads to a significant amount of additional liaison effort.

This advantage of the larger observatories is more or less offset by the fact that they involve much larger numbers of personnel. This tends to lead toward a breakdown of the personal working relationships and a greater formalization of the liaison. Realization of the advantage out 1 ined above for the observatories depends on the degree to which a personal, informal working relationship can be established between the experimenters and integration and operations groups, while maintaining the coordination necessary to ensure that the scientific objectives of any one experiment are not jeopardized by other experiments or the spacecraft.

The degree of involvement of the experimenter can 1 ie anywhere between two extremes. In the first, that of an experimenter who completely designs, builds, tests, integrates, and launches his own experiments, these efforts are performed within his own organization by personnei under his direct control. This condition is approached in the conduct of many balloon and sounding rocket programs. In the other extreme, the experimenter designs his experiment according to a carefully written set of interface specifications, delivers it to an integration crew at a central laboratory, and appears there occasionally to check the calibration. 
Operation after the launch can be divided into two similar extremes. In the former, the experimenter operates his own receivers and other ground equipment and carries his flight records to his own analysis group (or analyzes them completely himself). In the latter, a ground system is established and operated by a central laboratory, and the raw data are delivered to the experimenters in directly usable form.

Most actual satellite programs fall somewhere between these two extremes, and a considerable amount of liaison between the experimenters and other groups is necessary. The arrangement which is preferred by various experimenters depends to some extent on those individuais' personalities and the extent of their other responsibilities. Advantages are often cited for both extremes. The advocates of the completely selfcontained operation feel that they can directly influence all aspects of the project, and are less subject to the whims and shortcomings of a large number of strangers or near strangers. In addition, many of the experimenters located at universities feel it desirable to have their students become intimately familiar by direct experience with many different aspects of their projects, including the pre-launch integration and testing and in-flight operations.

The advocates of the central integration, testing, launching, and operations facility, feel that they have more time for their primary interest, the development of new experiments and analysis of their data. They feel that they are able to conduct a larger number of experiments and derive more results per unit time than experimenters who concern 
themselves with all of the technical details of the spacecraft subsystems, ground receiving stations, etc. Since these two different approaches stem from strongly felt genuine differences of opinion on the parts of the members of the scientific community, it is doubtful whether a single method of operating satellite programs can or should ever be found. Presently there seems to be a continued acceptance of both general methods of operation as evidencedby the recent approval of the university small sateilite program on the one hand and the continued scheduling of the large observatories on the other.

ORBITAL REQUIREMENTS

The smali satelite offers a definite advantage in meeting the requirements of the experiments for specific individual orbits. A satellite containing a single experiment can be launched into an optimum orbit for that experiment. A large observatory carrying many experiments must be launched into an orbit which best meets the needs of as many experiments as possible. It will, in general, be optimum for less than the entire set of experiments. In the 1 imit, as the number of spacecraft per year became very large, this distinction would disappear, since large numbers of experiments would require the same orbit. It is doubtful whether this condition will ever be reached. It is true, however, that there are now sufficiently large numbers of experiments requiring roughly similar orbits to justify the use of multi-experiment observatories. But it is also true that small satellites are necessary for some experiments requiring specialized orbits. 


\section{UTILIZATION OF GROUND FACILITIES}

The ground facilities, including tracking and data acquisition stations, control centers, orbital computation facilities, and communications and data relay links can be used more efficiently for larger observatories. It requires considerably more equipment and personnel to acquire data from a number of small satellites than from a single large one containing the same experiments. The same holds for tracking and orbit computation. In fact, it may be easier to compute an accurate orbit for a single large satellite than for a single small one, since the large one can include higher powered and higher performance tracking beacons and transponders. The control center and communications links can be operated for a large observatory with less expenditure of resources than for an equivalent number of small satellites, assuming they have comparable command and other operational capabilities.

The data processing facility utilization is not quite as straightforward. It may be argued that the data processing rate would depend only on the telemetered bit rate, in which case a ranking in terms of satellite class would not be meaningful. But this neglects editing, tape evaluation, and computer loading times, which are more nearly proportional to the number of data acquisition station recordings than to the number of telemetered data bits. Therefore, the observatories with their high data rates also offer an advantage in the utilization of the data processing facilities. 


\section{RELIABILITY}

The electronic subsystems complexity is higher for large observatories than for small satellites. Thus, it might seem that a small satellite would operate longer than an observatory. And it might appear likely that more data would be obtained before failure from a number of small sateliites than from the same number of experiments in an observatcry. This seeming advantage of the small satellites is offset by several factors.

1. The more complex observatory subsystems can be divided ints patially or completely redundant subsystems, such that a large fraction of the goals can be achieved even if a number of failures occurs.

2. A larger observatory can carry a large capability command system, allowing the correction of many problems as they occur.

3. Since an observatory is not tailored to each load of experiments to as large a degree as the small satellites, its subsystems can be used repeatedly without essential modification. The reliability of a system increases as it evolves through a long use history, as weaknesses are corrected and as production, testing, and operational personnel become more familiar with it.

4. Since the observatory systems are designed for repeated use, more effort can be expended per unit complexity in their development.

These factors act in such a manner that there is no clear-cut reliability differenze at present between individual small and large observatories. There is some indication, however, that the observatories may emerge as the more reliable of the two. This is based primarily on a 
comparison of OGO-I with the Explorer satellites. Table III lists the comparative reliability data for OGO-I and Explorer XII whose performance is typical of the small satellite class.

Table III

Reliability Comparison

No of Equivalent Parts

Saiel1ite

Identification

Excluding

Including

Useful

Experiments

Experiments

Life

$1961-$ Y1, Exp.XII

2,900

6,400

(days)

1954 - 54A, OGO-I

16,600

36,600

.212

$201 *$

*As of 1 May 1965 and still operating.

In Table III, the equivalent number of parts represents a parts count which has been adjusted for redundancy. Useful life represents the time period within which the satellites have provided significant scientific information. It is interesting to note that OGO-I has experienced fallure of two booms to deploy, failure to track the earth and sun because of the boom failure, failure of one of the two data handling systems, and cessation of oscillation of an inverter (subsequently restarted), but that it continues to return large volumes of scientificaliy significant data. This is attributed directly to the large amount of system redundancy and to the use of the high capacity radio command system which has permitted at least partial compensation for many of the failures. 


\section{CONCLUSIONS}

The discussions above have indicated that each of the two main classes of scientific earth satellites has distinct advantages. These are summarized in Table IV. A cross in one column indicates that that satellite class generally has an advantage in the category listed. Where neither class has a clear advantage, a dash is shown. Question marks indicate that insufficient information exists to properly evaluate the category.

Table IV

\section{Category}

Smali

Sata11ite
Large Observatory

1. Ability to laurch large or heavy experiments, or many related experiments

$\mathrm{X}$

2. Applicability of simple orientation techniques

3. Applizability of high capability active orientation techniques

4. Information rate

5. Availability of electrical power

6. Experiment interference

7. Ease of integration

8. Program coordination, testing, scheduling

9. Ability to meet orbital requirements of all experiments

10. Jtilization of ground facilities

11. Reliability
$\mathrm{X}$

$\mathrm{X}$

$\mathrm{X}$

$?$

$\mathrm{X}$

$\mathrm{X}$

? $\mathrm{x}$

$?$

? 
It is therefore obvious that the long range space science program will continue to require the use of both the small satellites and large observatories in order to meet the needs of the large variety of experiments and researchers. 


\section{FIGURE CAPTIONS}

Figure 1. The number of small scientific satellites successfully launched as a function of weight. All space science earth orbiting satellites launched to the end of 1964 are included for which weights were announced. Vanguard I. and six TRS satellites, each weighing less than $2.5 \mathrm{~kg}$, are included although they are not normally included in the Explorer class. Communication, navigation, and weather satellites are not included.

Figure 2. The IMP-I (Explorer XVIII) PFM telemetry data format. This system is a modified tone burst-blank system in which the tone frequency contains the information. One burst-blank period $(0.32 \mathrm{sec})$ makes one channel. Sixteen channels $(5.12 \mathrm{sec})$ makes one frame. Sixteen frames $(81.92 \mathrm{sec})$ makes one sequence. The tone burst is normally used to send digital information ( 8 quantizing levels or 3 binary bits per burst) or analog samples ( $1 \%$ accuracy). In six of the frames the blanks are eliminated so that analog quantities can be transmitted continuously for 4.80 second periods.

Figure 3. The OGO-I main frame PCM telemetry data format. The number in the upper left hand corner of each box denotes the word number. The number in the center of the box designates the OGO-I experiment number using that word. The letter following some experiment numbers indicates whether the analog or digital 


\section{FIGURE CAPTIONS (continued)}

option was chosen. Nine binary bits make one word. A total of 128 words makes one frame. And 128 frames (128 subcommutator words) make one sequence. Bit rates are 1000 , 8000 , or 64,000 bits per second $(1,8$, or 64 sequences per 0.868 second). 


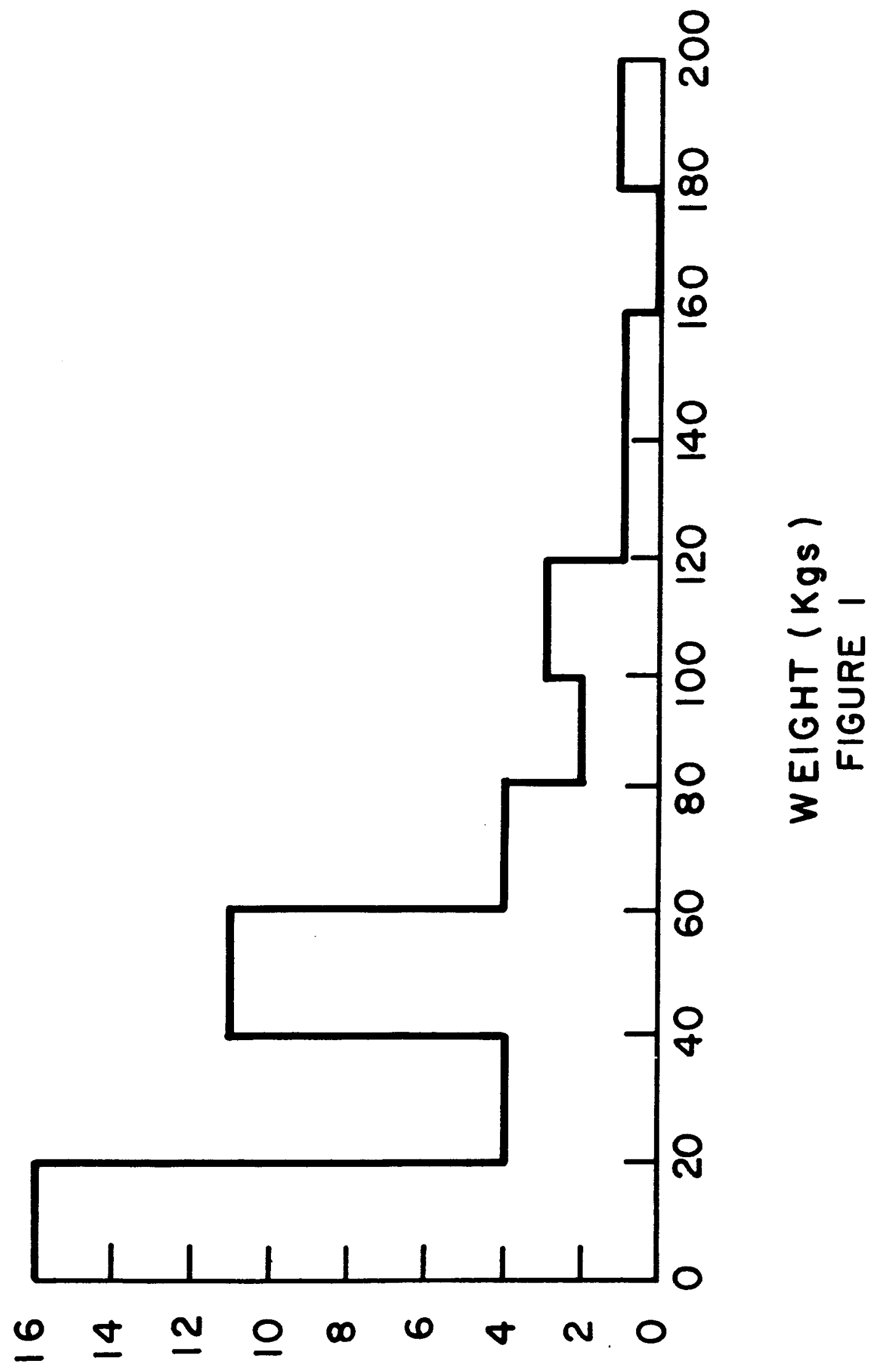

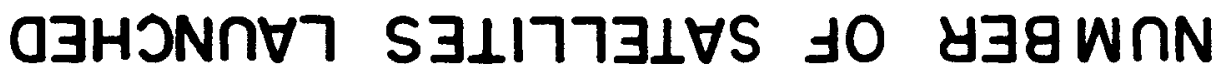


CHANNEL NUMBER

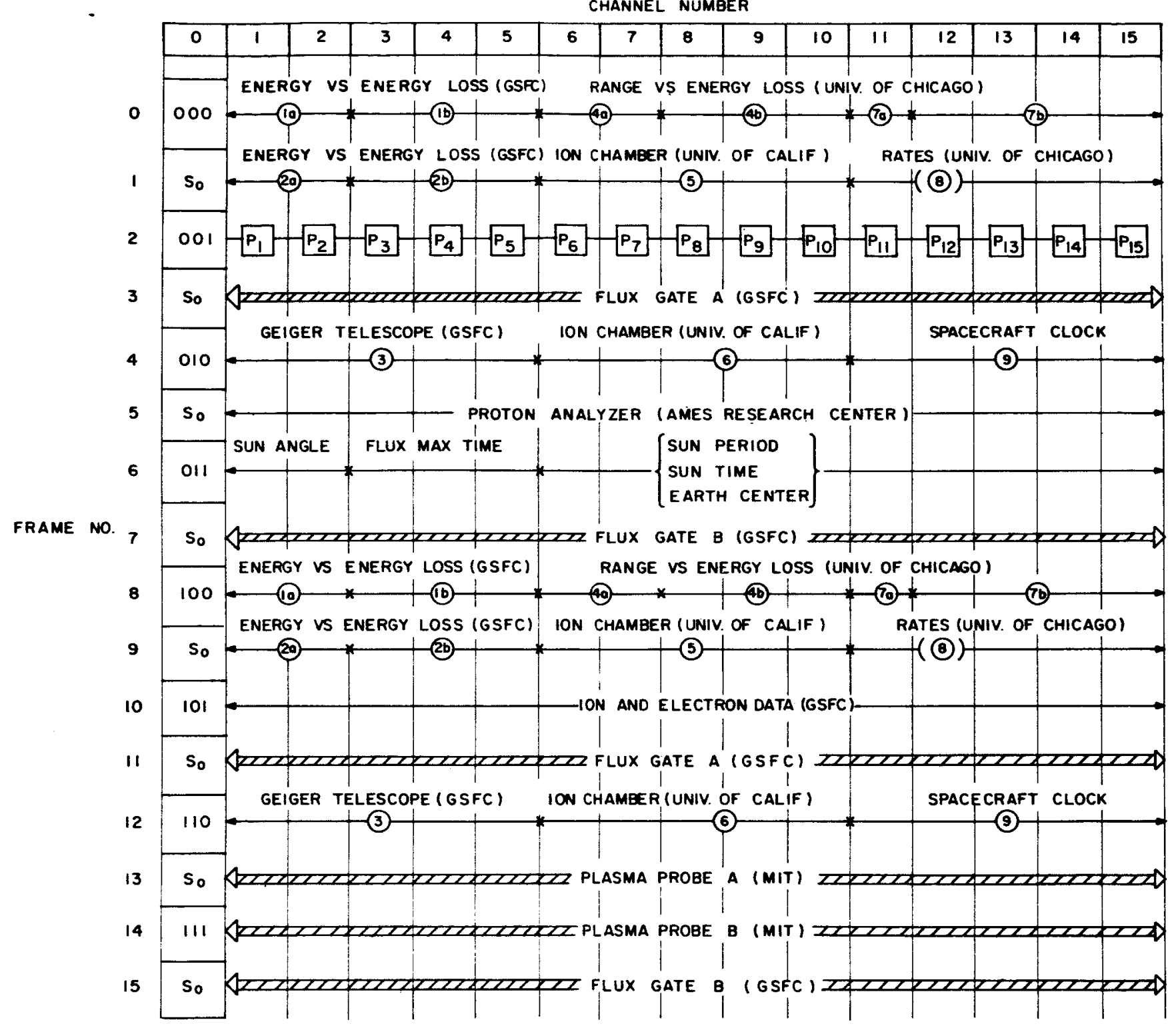

NOTES :

- Dig- Digital SCALER IDENTIFICATION-I Digital buRst PER CHANNEL

ATOE ANALOG READ OUT-CONTINUOUS 4.BO SECOND BURST

$P_{m}$ - PERformanCE PARAMETER IDENTIFICATION - I ANALOG SAMPLE PER CHANNEL

So - SYNC OSC. $4.5 / 16 \mathrm{KC}$

DIGITAL BURSTS ( 3 BITS) TAKE 0.16 SEC FOLLOWING A 0.16 SEC BLANK

ANALOG SAMPLES TAKE 0.16 SEC FOLLOWING A 0.16 SEC BLANK

SYNC CHANNEL BURSTS TAKE 0.24 SEC FOLLOWING A 0.08 SEC BLANK

\section{IMP - I TELEMETRY FORMAT FIGURE 2}




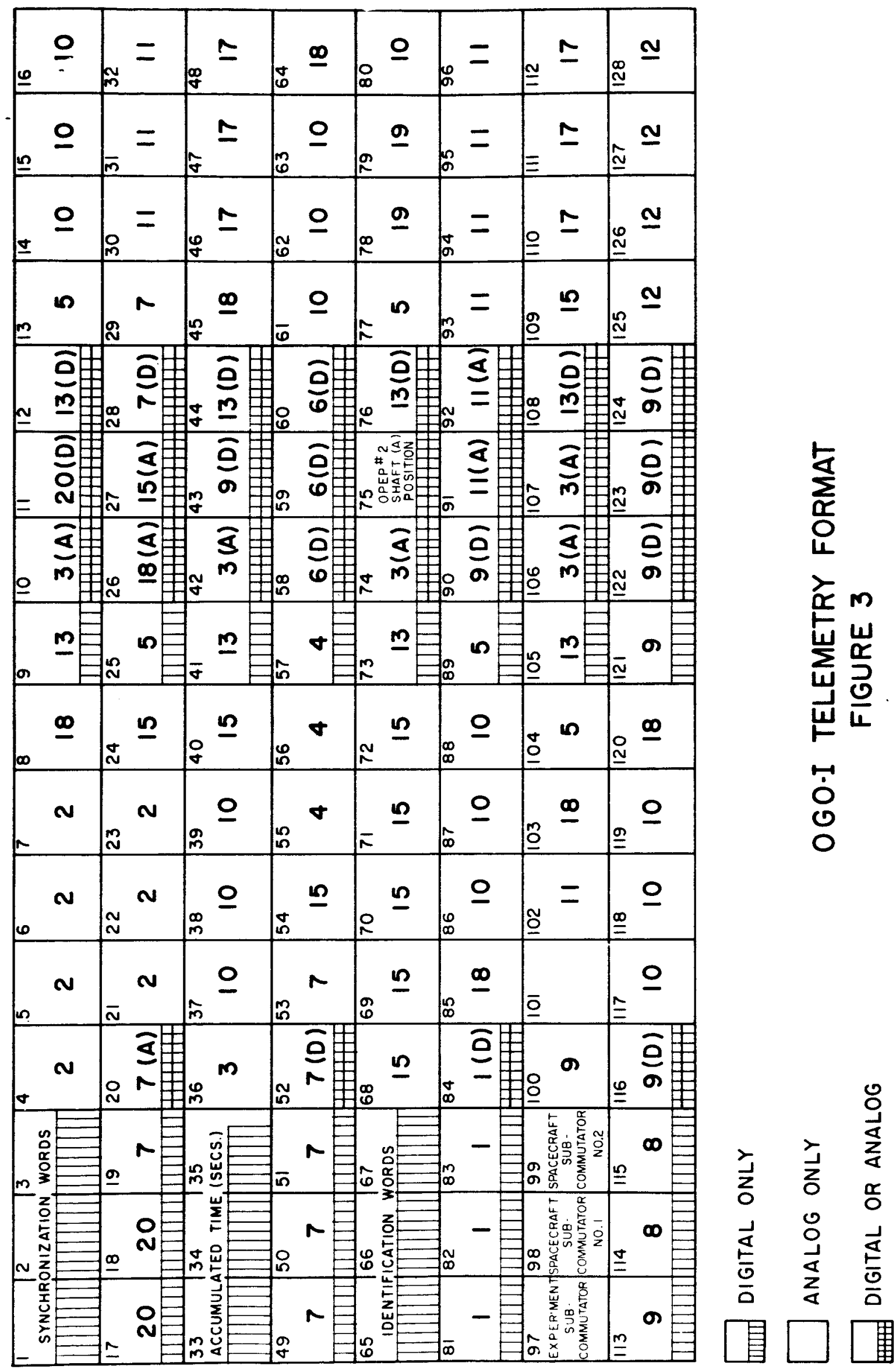

